\title{
Private pain - identifying gaps in the management of chronic pain patients in private hospitals: A case study
}

Follow this and additional works at: https://www.journal.acorn.org.au/jpn

Part of the Health Services Administration Commons, Health Services Research Commons, Perioperative, Operating Room and Surgical Nursing Commons, and the Surgery Commons

(c) $\underset{\mathrm{BY}}{(\rightarrow)}$

This work is licensed under a Creative Commons Attribution 4.0 License.

\section{Recommended Citation}

Gale, Johanna (2018) "Private pain - identifying gaps in the management of chronic pain patients in private hospitals: A case study," Journal of Perioperative Nursing: Vol. 31 : Iss. 3 , Article 5.

Available at: https://doi.org/10.26550/2209-1092.1036

https://www.journal.acorn.org.au/jpn/vol31/iss3/5

This Article is brought to you for free and open access by Journal of Perioperative Nursing. It has been accepted for inclusion in Journal of Perioperative Nursing by an authorized editor of Journal of Perioperative Nursing. 
Author

Johanna Gale

MN (Anaesthetic and Recovery), RN jgale34@optusnet.com.au

\title{
Private pain - identifying gaps in the management of chronic pain patients in private hospitals: A case study
}

\begin{abstract}
This is a case study of a patient who experiences chronic pain and was admitted for an acute surgical procedure in a private hospital. It illustrates how communication and teamwork can be overlooked within the multidisciplinary focus when jointly caring for this group of patients within the public and private sectors. This case study offers a nurse-led approach to improving individualised health care for chronic pain patients while preventing gaps in health care.
\end{abstract}

Keywords: Chronic pain, preoperative assessment, communication, pain team, private

\section{Introduction}

During eight years of nursing in recovery, I have encountered many patients who have a history of chronic pain and require acute postoperative pain management. Often these patients regain consciousness in recovery with severe pain and this is the first indication that there is an issue. After an extended period of receiving large doses of intravenous opiates in recovery, the patient is transferred to the ward. Pain management then becomes the focus of nursing care for the patient, who may already have a history of opioid tolerance. The length of stay increases beyond the estimated time agreed by surgeons, and private health funding for patients is limited to 'disease type.' The delay in referral of chronic pain patients to the pain team can result in negative effects on factors such as mobility, psychosocial wellbeing and motility?

The areas for improvement during hospitalisation examined in this case study will focus on the preoperative stage. For reasons of confidentiality the patient is referred to as 'Ann'. The aim is to propose a solution to enhance the early coordination of multidisciplinary care and improve communication among teams. It is hoped that this will provide a streamlined package of individualised care for patients with chronic pain.

\section{Definition of chronic pain}

The definition of chronic pain is pain lasting for three or more consecutive months during a recent six-month period $^{2}$. Sufferers of chronic pain commonly describe their pain as 'burning', 'sharp', 'shooting' and 'electric', which formulate a diagnosis of neuropathic pain ${ }^{3}$. Many surgeons have appeared to be noticeably perplexed regarding the cause of extreme pain due to a lesser or absent organic explanation for it. The previous definition of neuropathic pain was specific to the 'peripheral or central nervous system' and stated the cause of pain was due to a 'dysfunction'. Following collaboration and analysis of newly diagnosed pain disorders by global 
pain specialists, the new definition is, "pain caused by a lesion or disease of the somatosensory system ${ }^{5}$. This gives a much broader sense of what neuropathic pain can mean for some patients. It is less compartmentalised to the central and peripheral nervous system and therefore has broadened the potential for patients who have experienced long-term pain involving nerve fibres, such as chronic cystitis, to obtain an appropriate diagnosis leading to more suitable pain management ${ }^{5}$.

\section{Setting}

The case study is from an internationally recognised private, 270-bed metropolitan teaching hospital that provides acute medical and major surgical care. It employs 350 consultants who not only perform the latest procedures but are also pioneers within their specialty. The acute pain service was launched in 2014 and aims to provide globally recognised pain management techniques that will benefit in-patients. The service is anaesthetic-led and assists in the referral of acute surgical patients whose pain may require further management. Referrals are made by surgeons or physicians. The campus is shared by the public hospital where many of the same surgeons and anaesthetists provide treatment.

\section{Case study history}

This case study involves 'Ann', a woman who presented to the emergency department with a history of chronic lower back pain radiating down the left leg with associated numbness. Ann was admitted for an L5/S1 microdiscectomy the following day in the public hospital. While using an oral multimodal analgesic approach, the anaesthetist prescribed a patient-controlled analgesia (PCA) method and a continuous ketamine infusion. Ann was seen by the acute and chronic pain team in the hospital as her pain was not adequately alleviated postoperatively and as routine follow-up for the use of a PCA in this public hospital. Her subcutaneous ketamine and PCA were gradually ceased and oral analgesia titrated. The team recommended that all opiates be weaned within six weeks by her general practitioner. The hospital pain team suggested a community referral to the chronic pain team should the pain continue. Four months later she was readmitted by the same neurosurgeon, after consultation, to the private hospital on campus with severe lumbar pain.

\section{Preoperative assessment}

Surgery was scheduled for the following day for a redo-lumbar microdiscectomy due to evidence of re-occurring disc prolapse. Ann's regular pain regimen remained inclusive of Amitriptyline, Lyrica ${ }^{\circledR}$, Targin ${ }^{\circledR}$ and breakthrough Endone ${ }^{\circledR}$. She had no history of alcohol or recreational drug abuse or anxiety and depression. Patients with a psychological history can experience increased episodes of pain and, in turn, pain can intensify psychological symptoms ${ }^{3}$. It is important to gain this information in the assessment period to pre-empt this cascade of events from occurring. There was no evidence of an out-patient referral to the chronic pain team. Ann's pain score, using the verbal numerical pain score, was $6 / 10$. The verbal numerical pain score is easy to use for patients, and it is subjective ${ }^{6}$. I have discussed this with colleagues and collectively we consider a score four or more to indicate moderate to severe pain. The verbal numerical pain score when used alone may have a negative effect because our judgement may be allowed to influence the experience of the patient?. To avoid this influence, I use the verbal numerical pain score in conjunction with questions about how the patient would treat their pain if at home, thus preventing me from manipulating the score based on my experiences of pain. I had one patient who stated his pain was $6 / 10$ but he would only take paracetamol to control it. A phenomenological study of nurses' insight into pain scores supports this practice by establishing that additional information is required to provide appropriate management ${ }^{8}$. In Ann's case, a score of 6/10 had been common for her and she described it as moderate.

In the assessment stage, it is necessary to include a description of the patient's pain. The documentation of type and frequency of pain is required to differentiate between nociceptive and neuropathic pain. A correct assessment of the type of pain gives the patient the best chance of receiving appropriate pain management. One measurement chart to assess pain is the Neuropathy Pain Scale which includes descriptors, intensity and emotional factors to gauge how pain affects a patient's overall wellbeing ${ }^{9}$. The assessment of neuropathic pain can assist the nurse in investigating signs of acutely heightened pain (hyperalgesia) or the existence of pain caused by a nonpainful provocation such as touch (allodynia) ${ }^{6}$. With a description of the pain, it is vital to document the patient's daily medication regime as this indicates the significance of pain to the individual. In this case, Ann had withheld all her usual analgesics. It is important for patients who use analgesics regularly to continue their normal routine preoperatively. It is hard for the anaesthetist to recompense missed doses and managing the patient's pain will predictably become a challenge for recovery staff ${ }^{10}$. 
The anaesthetist recognised that Ann was 'opioid tolerant' during her preliminary examination. Opioid tolerance is a foreseeable response following a prolonged period of self-administration and requires the treating physician, in this case the anaesthetist, to administer greater amounts to achieve a level of efficacy ${ }^{11}$. Opioid tolerance is not to be confused with addiction or dependence. As nurses, we must remind ourselves of the difference. Patients with chronic or 'persistent' pain hold a significant amount of knowledge about their analgesic program and expect the same of health care professionals ${ }^{12}$. The recent renaming of 'chronic' to 'persistent' pain has come about to counteract the negative stigma associated with chronic pain. All of us have heard at least one nurse in our career saying 'Oh no, not another chronic pain patient'. Nurses who are armed with the knowledge to assess and appropriately treat those patients whose pain can escalate will help those patients significantly, leading to greater patient satisfaction ${ }^{13-15}$.

Before admission, Ann was prescribed a combination of medications that have a number of actions. The anti-convulsant Lyrica ${ }^{\circledR}$ interrupts messages between the brain and damaged nerves. In low doses, the antidepressant Amitriptyline prevents the reabsorption of serotonin and noradrenaline into the nerve, lessening pain sensation and having a positive emotional effect. Opioids, such as Targin ${ }^{\circledR}$ and Endone ${ }^{\circledR}$, join to the opiate receptors found in the central and peripheral nervous system, limiting the number of pain signals sent from the nervous system to the brain ${ }^{3}$. The International Association for the Study of Pain (IASP) has provided guidelines to include this combination of therapies based on research that they have greater efficacy than the use of stand-alone opioids ${ }^{16,17}$.

In conjunction with the type of analgesia patients take, the amount, frequency and efficacy should be assessed along with any nonpharmacological methods used. By gaining additional information, a management plan can be discussed with the anaesthetist. If a pain team is present, they should be contacted to discuss the patient's case. In this case, Ann was known to the public acute pain team and, at present, the private hospital has a physician-only referral system. The ability for nurses to refer to other nurse-led teams has been shown to be an active and fast solution for managing patients' holistic needs ${ }^{18}$.

The identification of chronic pain at the assessment stage can trigger the need for ongoing evaluation of pain with an emphasis on the tools to assess chronic pain. Communicating this requirement to the anaesthetic nurse as well as the acute pain team initiates the process of planning individualised care. Verbal and written documentation highlighting the patient's analgesic history and progressive pain management to the multidisciplinary team promotes a high level of patient care and is a responsibility of all health care workers, authorised by the Clinical Excellence Commission ${ }^{19}$.

\section{Perioperative care}

As was the case with the first surgery, the anaesthetic analgesic protocol included a multi-modal approach of intravenous opiates, ketamine, paracetamol and dexamethasone. The use of the surgical safety checklist at the beginning of the operation would have proved an appropriate opportunity for members of the anaesthetic and surgical team to discuss post-operative pain management. Communication should be initiated either during 'time out' when the anaesthetist discusses patient concerns or during the 'sign out' stage when the surgeon and anaesthetist or anaesthetic nurse discuss potential management issues in the post-anaesthesia care unit ${ }^{20}$.

Due to the repetition of surgery within a short period, there is the potential for increased postoperative pain. Increased pain may be caused by the incision of scar tissue, over-sensitisation of the sciatic nerve root because of involved manipulation or a tear to the dura ${ }^{21}$. Since Ann's previous surgery her mobility had reduced and her body mass index was higher than average. These factors can lead to preoperative muscle and joint stiffness, resulting in difficulty with rehabilitation during the post-operative period. The use of increased doses of intravenous opiates in the immediate postoperative period causes sedation which delays physiotherapy.

\section{Post-operative recovery}

Post-extubation Ann was in severe pain, verbalising a numerical pain score of $9 / 10$. The pain was similar to the preoperative nerve pain in association with surgical incision pain. Due to the lack of documented communication of Ann's chronic pain history, the recovery team had to use pharmacological and nonpharmacological methods to control the pain and offer reassurance to the patient. Intravenous bolus doses of fentanyl were given, totalling 200 micrograms before the commencement of a fentanyl PCA. Ann's pain score was 6/10 at its lowest rating. On the ward, pain management became the focus of care for the following four days, balancing opiate induced sedation with an attempt at daily physiotherapy. Reduced mobility and lowered mood had begun to impinge on recovery. A magnetic resonance imaging scan (MRI) was performed to identify the cause of ongoing 
neuropathic pain in the left thigh. There was no evidence of nerve compression or dural tear which led the surgical team to conclude Ann was acutely sensitive to surgical intervention which had been otherwise uncomplicated.

Though the surgical team had performed daily rounds, there was little evidence of documented pain assessment in nursing notes other than the first 24 hours when the PCA was in use. Ann never verbalised a score of less than six. The assessment and documentation of pain have been related to improvement in patients' pain management ${ }^{22}$. In a United Kingdom audit which evaluated pain as the 'fifth vital sign', it was found that patients with chronic pain had different needs to those with acute pain $^{22}$. The audit also discovered that documenting scores was considered to be less necessary than the pain team recognising the need for early treatment planning 22 .

On day four, the surgeon referred the patient to the acute pain team, which in the private hospital is anaesthetist based. As the anaesthetists also have their operating lists, there is the possibility of delay between referral and assessment by the pain specialist. On this occasion, Ann was seen promptly. The plan was to stop Targin and introduce Tapentadol which has been proven in clinical trials to be more effective than Oxycodone while having fewer gastrointestinal side-effects, such as nausea and constipation, and being easier to be weaned from due to its less addictive properties ${ }^{23}$. The possibility of withdrawal symptoms did occur along with apathy. Tramadol was prescribed for breakthrough pain and Endone was limited to one a day and reserved for only the most severe pain. The anaesthetic pain specialist reassured
Ann that the new pain management and the increase in mobility would slowly occur. The discussion of pain and timelines for recovery can alleviate false expectations, particularly with patients who are enduring chronic pain ${ }^{24}$. The introduction of Clexane injections, to prevent venous thromboembolism (VTE) due to Ann's reduced mobility, and steroids to reduce nerve inflammation were commenced the next day. From day five to ten slow recovery was made. The pain team devised a plan for Ann's GP to wean her from Tapentadol; however, she was administered, upon request, a total of $15 \mathrm{mg}$ of Oxycodone incrementally during the night before discharge (daily allowance of $15 \mathrm{mg}$ ). Her discharge home on day ten was eight days longer than planned and financially determined by private health funds. Chronic pain is not considered a separate disease entity and therefore is not funded independently. To date, the National Pain Strategy group continues to seek acknowledgement that chronic pain is identified and treated as an isolated disease by the federal and state governments ${ }^{2}$.

\section{Clinical recommendations}

Given Ann's previous surgical history and difficulty with post-operative pain management, a proposal for immediate referral to the pain team at the pre-admission stage may have initiated an early pain management plan and reduced the length of stay. Communication between the surgical team and acute pain team from the public and the private hospital could be improved by the sharing of data of chronic pain patients to prevent this gap in treatment. Educating nursing staff to identify neuropathic pain and introducing an assessment chart could provide additional information for the multidisciplinary team members including physiotherapists and pharmacists. Confirming pain assessment as the 'fifth vital sign' has been shown to improve documentation and provide increased patient satisfaction. Lastly, the addition of a pain nurse to the pain team may serve as a more cost-effective resource for early recognition of patients who are being undermanaged and require additional support.

\section{Conclusion}

In the author's experience, this case study is not an isolated occurrence. Each discipline worked well to accommodate the patient's needs but collectively the team lacked cohesion when promoting holistic care. Patients with chronic pain continue to be treated the same as those with acute pain when admitted for acute surgical intervention. This case study has highlighted a gap in documentation and communication between multidisciplinary team members thus hindering individualised care for chronic pain patients.

\section{References}

1. Herr K. Pain assessment and strategies in older patients. J Pain. 2011;12(3):S3-S13.

2. National Pain Strategy (Internet). Melbourne: Royal Australasian College of Physicians; 2009 Oct (updated 2015; cited 2016 September 28). Available from: www.anzspm.org.au/c/anzspm?a= sendfile\&ft $=p \&$ fid $=1320268502 \&$ sid

3. Macintyre PE, Schug SA. Acute pain management: A practical guide. Edinburgh, Scotland: Saunders Elsevier; 2007.

4. Merskey H, Bogduk N. Classification of chronic pain. Seattle, WA: IASP Press; 1997.

5. Jensen $T$, Baron R, Haanpaa M, Kalso E, Loeser J, Rice A et al. Pain. 2011;152(10):22042205.

6. Pasero C, McCaffery M. Pain assessment and pharmacologic management. St. Louis: Mosby; 2011.

7. Hartrick CT, Kovan JP, Shapiro S. The numeric rating scale for clinical pain measurement: $A$ ratio measure? Pain Pract 2003;3(4):310-316. 
8. Wikström L, Eriksson K, Årestedt K, Fridlund B, Broström A. Healthcare professionals' perceptions of the use of pain scales in postoperative pain assessments. Appl Nurs Res 2014;27(1):53-58.

9. Galer BS, Jensen MP. Development and preliminary validation of a pain measure specific to neuropathic pain. Neurol 1997;48(2):332.

10. Dykstra KM. Perioperative pain management in the opioid-tolerant patient with chronic pain: An evidence-based practice project. J Perianesth Nurs 2012;27(6):385-392.

11. Schug SA, Palmer GM, Scott DA, Halliwell $R$, Trinca J. APM:SE Working group of the Australian and New Zealand College of Anaesthetists (ANZCA) and Faculty of Pain Medicine (FPM). Acute pain management: Scientific Evidence ( $4^{\text {th }}$ edition), Melbourne; ANZCA \& FPM; 2015.

12. Benfari RN. Management of the patient with chronic pain. Crit Care Nurs Clin North Am 2015;27(1):121-129.

13. Ravaud P, Durand-Stocco C, Keïta H, Desmonts JM, Mantz J, Porcher R. Randomised clinical trial to assess the effect of an educational programme designed to improve nurses' assessment and recording of postoperative pain. Br J Surg 2004;91(6):692-698.
14. Guardini I, Talamini R, Fiorillo F, Lirutti M, Palese A. The effectiveness of continuing education in post-operative pain management: Results from a follow-up study. I Contin Educ Nurs 2008;39(6):281-288

15. Abdalrahim MS, Majali SA, Stomberg MW, Bergbom I. The effect of post-operative pain management program on improving nurses' knowledge and attitudes toward pain. Nurse Educ Pract 2011;11:250-255.

16. Costello M, Greenberg H, Bell C, TrowersSmith J. Management of the hospitalized patient experiencing acute on chronic pain: A case study. Pain Manag Nurs 2013;14(4):E268-E73.

17. Brown CA, Richardson C. Nurses in the multi-professional pain team: A study of attitudes, beliefs and treatment endorsements. Eur J Pain 2006;10(1):13-18.

18. Courtenay M, Carey N. The impact and effectiveness of nurse-led care in the management of acute and chronic pain: A review of the literature. J Clin Nurs 2008;17(15):2001-2013.

19. Clinical Excellence Commission. Clinical handover - standard key principles [Internet]. NSW: NSW Government; 2009 [updated 2014 September; cited 2016 September 29]. Available from: www1.health.nsw.gov.au/PDS/pages/doc. aspx?dn=PD2009_060.
20. World Health Organisation (WHO). WHO surgical safety checklist [Internet]. Geneva: WHO; 2008 (updated 2008 June; cited 2016 September 30). Available from: www.who.int/ patientsafety/safesurgery/ss_checklist/en.

21. Dae-Jung C, Je-Tea J, Sang-Jin L, YoungSang K, Han-Jin J, Bang Y. Biportal endoscopic spinal surgery for recurrent lumbar disc herniations. Clin Orthop Surg 2016;8(3):325-329.

22. Purser L, Warfield K, Richardson C. Making pain visible: An audit and review of documentation to improve the use of pain assessment by implementing pain as the fifth vital sign. Pain Manag Nurs 2014;15:137-142.

23. Afilalo M, Etropolski MS, Kuperwasser B, Kelly K, Okamoto A, Hove IV et al. Efficacy and safety of Tapentadol extended release compared with Oxycodone controlled release for the management of moderate to severe chronic pain related to osteoarthritis of the knee. Clin Drug Investig 2010;30(8):489-505.

24. MacLellan K. Postoperative pain: Strategy for improving patient experiences. J Adv Nurs 2004;46(2):179-193.

\section{International volunteer and teaching grant recipient}

ACORN wishes to support its members and the broader perioperative community by offering financial support for members who are intending to do voluntary work or teach perioperative nursing skills overseas.

The 2018 recipient of this grant is Ross Mcllwraith. Ross will spend three months as a volunteer scrub/scout nurse on the hospital ship, Africa Mercy. The ship has five operating theatres on board and is part of the Mercy Ships international charity that provides humanitarian aid around the world. The grant will cover Ross's travel expenses and some expenses while on board. 